\title{
BMI status of children in the CHANGE! Project and its association with the consumption of 'positive marker' and 'negative marker' foods
}

\author{
G. L. Warburton ${ }^{1}$, E. Mahon ${ }^{1}$, I. G. Davies ${ }^{1}$, R. Gobbi ${ }^{3}$, K. Mackintosh ${ }^{4}$, S. J. Fairclough ${ }^{2}$ \\ and J. C. Abayomi ${ }^{1}$ \\ ${ }^{1}$ Faculty of Education, Community and Leisure, Liverpool John Moores University, Liverpool, UK, ${ }^{2}$ Research Institute \\ for Sports and Exercise Sciences, Liverpool John Moores University, Liverpool, UK, ${ }^{3}$ Faculty of Science and Social \\ Science, Liverpool Hope University, Liverpool, UK and ${ }^{4}$ Applied Sports Technology Exercise and Medicine Research \\ Centre (A-STEM), College of Engineering, Swansea University, Swansea, UK
}

Currently, around a third of Year 6 children are reported to be overweight or obese ${ }^{(1)}$. The association between diet and obesity is wellevidenced but it is the association with patterns of dietary intake, in particular the intakes of certain nutrients or groups of foods, which may be influencing childhood obesity rates ${ }^{(2)}$. The CHANGE! Project (Children's Health, Activity, and Nutrition: Get Educated!), involving Year 6 children ( $n=290$ total; $n=138$ intervention; $n=152$ control) in Wigan, aimed to promote the benefits of healthy eating and physical activity, by making small behavioural changes to lifestyle. Data regarding the food intake of the participants was collected at three data collection points throughout the school year, using a validated 24-hour recall questionnaire ${ }^{(3)}$. Subsequent classification of particular types of foods into 'positive marker foods' (PMF) and 'negative marker foods' (NMF) as a percentage of possible intakes of these types of foods, allowed for comparisons to be made between data collection points and the grouped BMI status of children. Overall, there was no significant difference between BMI status of children and their reported intakes of PMF and NMF between each data collection point. However there was a significant difference (Independent samples $t$-test; $p=0.006$ ) at follow-up between the intakes of PMF and underweight (UW) and normal weight (NW) children, and overweight (OW) and obese (OB) children, with the OW and OB children reportedly consuming approximately $4 \%$ more PMF at this data point.

\begin{tabular}{|c|c|c|c|c|}
\hline & & Baseline & Post-intervention & Follow-up \\
\hline NMF (\%) & $\begin{array}{l}\mathrm{UW}+\mathrm{NW} \\
\mathrm{OW}+\mathrm{OB}\end{array}$ & $\begin{array}{l}31.46 \\
31.93\end{array}$ & $\begin{array}{l}31.17 \\
28.54\end{array}$ & $\begin{array}{l}28.21 \\
24.59\end{array}$ \\
\hline $\operatorname{PMF}(\%)$ & $\begin{array}{l}\mathrm{UW}+\mathrm{NW} \\
\mathrm{OW}+\mathrm{OB}\end{array}$ & $\begin{array}{l}29.42 \\
26.35\end{array}$ & $\begin{array}{l}29.87 \\
27.52\end{array}$ & $\begin{array}{l}26.63 * \\
30.78^{*}\end{array}$ \\
\hline
\end{tabular}

$\left({ }^{*} p=0.006\right)$.

These mean percentage values also indicate a general trend of a decrease for all children in the consumption of NMF, and a decrease in the consumption of PMF for UW and NW children, but an increase in PMF by the OW and OB children only, at follow-up. Further investigation revealed that the $\mathrm{OW}$ and $\mathrm{OB}$ children claimed to have consumed more fruit, vegetables, fish and water than the UW and NW children at follow-up. Although there is a recognised association between obesity and diet, with reference to particular food groups, further evidence is required to determine the effect of continued exposure to those food groups which may be contributing to obesity in children.

1. Health and Social Care Information Centre. (2013) Statistics on Obesity, Physical Activity and Diet: England, 2013 [20th February 2013].

2. Jennings A, Welch A, van Sluijs EMF, Griffin S, Cassidy A. (2011) Diet Quality is Independently Associated with Weight Status in Children Aged 9-10 Years. The Journal of Nutrition 141(3), 453-9.

3. Johnson B, Hackett A, Roundfield M, Coufopoulos A. (2001) An Investigation of the Validity and Reliability of a Food Intake Questionnaire. Journal of Human Nutrition and Dietetics 14, 457-65. 